\title{
Técnicas de fixação de fraturas mandibulares: uma revisão integrativa da literatura
}

\author{
Techniques for fixing mandibular fractures: an integrative literature review \\ Técnicas para la fijación de fracturas mandibulares: una revisión integrativa de la literatura
}

Recebido: 16/12/2021 | Revisado: 23/12/2021 | Aceito: 30/12/2021 | Publicado: 07/01/2022

\author{
Maria Vitória Araújo Lima \\ ORCID: https://orcid.org/0000-0003-3855-7181 \\ Centro Universitário Tiradentes, Brasil \\ E-mail: maria.valima@ souunit.com.br \\ José Ronaldo Lourenço dos Santos Júnior \\ ORCID: https://orcid.org/0000-0001-6904-6216 \\ Centro Universitário Tiradentes, Brasil \\ E-mail: juniorlourenco10@hotmail.com \\ Maria Eduarda Alexandre Macedo Holanda \\ ORCID: https://orcid.org/0000-0001-9002-0678 \\ Centro Universitário Tiradentes, Brasil \\ E-mail: mariaaholanda@ hotmail.com \\ Maria Eduarda da Silva Nascimento \\ ORCID: https://orcid.org/0000-0002-8117-0102 \\ Centro Universitário Tiradentes, Brasil \\ E-mail: eduardaa_nas@hotmail.com \\ Nathalia Maria Silvia de Almeida \\ ORCID: https://orcid.org/0000-0003-0824-327X \\ Centro Universitário Tiradentes, Brasil \\ E-mail: natyalmeida78@hotmail.com \\ Carlos Marcelo dos Santos Pedrosa Filho \\ ORCID: https://orcid.org/0000-0003-2123-0107 \\ Centro Universitário Tiradentes, Brasil \\ E-mail: cmarcelodospf@ hotmail.com \\ Jessica Larissa do Nascimento Ursulino Barbosa \\ ORCID: https://orcid.org/0000-0002-7910-1343 \\ Centro Universitário Tiradentes, Brasil \\ E-mail: ursulinojes@outlook.com \\ Letícia Santos Costa \\ ORCID: https://orcid.org/0000-0001-7516-3937 \\ Centro universitário Tiradentes, Brasil \\ E-mail: leticosta30@gmail.com \\ Victor Silva Carvalho \\ ORCID: https://orcid.org/0000-0003-2594-2048 \\ Centro Universitário Tiradentes, Brasil \\ E-mail: victorcarvalho339@gmail.com \\ Vitor Antônio Nunes \\ ORCID: https://orcid.org/0000-0001-6025-2320 \\ Centro Universitário Tiradentes, Brasil \\ E-mail: vitorsp295@gmail.com
}

\begin{abstract}
Resumo
Diante das fraturas mandibulares existem algumas técnicas de fixação, dentre essas destaca-se a Técnica de Champy. Essas técnicas possuem o mesmo objetivo, o qual consiste na estabilização da fratura, devolvendo a função e saúde ao paciente. Para o desenvolvimento de uma conduta correta e obtenção de um bom resultado é imprescindível o conhecimento adequado das técnicas. O presente artigo tem como objetivo relatar a pesquisa realizada na literatura referente as técnicas de fixação. Tratou-se de uma revisão integrativa da literatura realizada através de buscas na base de dado LILACS. Os critérios de inclusão foram artigos nos idiomas português e inglês e publicados entre 2015 e 2021. Foram excluídas teses, livros, dissertações, resumos, trabalhos de conclusão de curso e estudos que não eram completos acerca do tema. Foram selecionados onze artigos, os quais cumpriam os critérios de inclusão e exclusão. $\mathrm{O}$ trauma é a maior causa de fraturas mandibulares, sendo estas as mais comuns quando comparadas as outras estruturas faciais. Seu diagnóstico e tratamento devem ser apropriados para poder restabelecer de maneira funcional e estética, pois tratamentos incorretos podem causar sequelas. As fraturas mandibulares aparecem com mais frequência na rotina dos bucomaxilofaciais, os quais realizam intervenções através de técnicas de fixação a fim de devolver a função, estética e anatomia da região abalada, com destaque para técnica de Champy.
\end{abstract}

Palavras-chave: Fixação de fratura; Mandíbula; Traumatismos mandibulares. 


\begin{abstract}
In the face mandibular fractures, there are some fixation techniques, among which the Champy Technique stands out. These techniques have the same objective, which is to stabilize the fracture, restoring function and health to the patient. For the development of a correct behavior and obtaining a good result, it is essential to have adequate knowledge of the techniques. This article aims to report the research carried out in the literature regarding fixation techniques. It was an integrative literature review carried out through searches in the LILACS database. The inclusion criteria were articles in Portuguese and English and published between 2015 and 2021. Theses, books, dissertations, abstracts, course completion papers and studies that were not complete on the topic were excluded. Eleven articles were selected, which met the inclusion and exclusion criteria. Trauma is the major cause of mandibular fractures, which are the most common when compared to other facial structures. Its diagnosis and treatment must be appropriate to be able to restore it in a functional and aesthetic way, as incorrect treatments can cause sequelae. Mandibular fractures appear more frequently in the routine of maxillofacials, who perform interventions through fixation techniques in order to restore the function, esthetics and anatomy of the affected region, with emphasis on the Champy technique.
\end{abstract}

Keyworsd: Fracture fixation; Mandible; Mandibular injuries.

\title{
Resumen
}

Ante las fracturas mandibulares, existen algunas técnicas de fijación, entre las que destaca la Técnica Champy. Estas técnicas tienen el mismo objetivo, que es estabilizar la fractura, devolviendo la función y la salud al paciente. Para el desarrollo de un comportamiento correcto y la obtención de un buen resultado, es fundamental tener un conocimiento adecuado de las técnicas. Este artículo tiene como objetivo informar de las investigaciones realizadas en la literatura sobre técnicas de fijación. Se trata de una revisión integradora de la literatura realizada a través de búsquedas en la base de datos LILACS. Los criterios de inclusión fueron artículos en portugués e inglés publicados entre 2015 y 2021. Se excluyeron tesis, libros, disertaciones, resúmenes, trabajos de finalización de cursos y estudios que no estuvieran completos sobre el tema. Se seleccionaron once artículos que cumplieron con los criterios de inclusión y exclusión. El trauma es la principal causa de fracturas mandibulares, que son las más comunes en comparación con otras estructuras faciales. Su diagnóstico y tratamiento debe ser el adecuado para poder restaurarlo de forma funcional y estética, ya que tratamientos incorrectos pueden provocar secuelas. Las fracturas mandibulares aparecen con mayor frecuencia en la rutina de los maxilofaciales, quienes realizan intervenciones mediante técnicas de fijación con el fin de restaurar la función, estética y anatomía de la región afectada, con énfasis en la técnica de Champy.

Palabras clave: Fijación de fractura; Mandíbula; Traumatismos mandibulares.

\section{Introdução}

A mandíbula é o único osso móvel da face, com isso, acaba sendo bastante atingida por traumas diretos ou indiretos decorrentes de acidentes automobilísticos e durante atividade física, queda da própria altura e violência. Representando, aproximadamente, dois terços de todas as fraturas que ocorrem na face. Estaticamente falando, a estudos que revelam que é o osso com maior incidência de fraturas na face e que gênero masculino apresenta maior freqüência de acometimento (Oliveira et al., 2021; Dantas et al., 2018; Flandes et al., 2019). Os principais sinais e sintomas observados em pacientes com fratura mandibulares assimetria facial, desnível oclusal, mobilidade dos fragmentos ósseos, hematomas, dor e trismo (Alencar et al., 2015; Rantes et al., 2019).

As fraturas mandibulares têm maior ocorrência após traumas do complexo maxilomandibular e a sua maior prevalência é na região goníaca. O tratamento apropriado tem como finalidade restituir aspectos estéticos e funcionais (Luciano et al., 2018). Do ponto de vista anatômico, as regiões que mais apresentam fraturas são ângulo, côndilo, sínfise, corpo mandibular, ramo e processo coronóide. Uma divisão bastante funcional é a do padrão de fratura, sendo, simples, única, composta, complexa, múltipla ou galho verde e favorável ou desfavorável quanto à sua inserção no músculo (Dantas et al., 2018).

As principais formas e técnicas adequadas de fixação para os casos de fratura mandibular, dentre elas destacam-se a Técnica de Champy, a qual consiste em uma redução intraoral, aplicando uma placa na borda superior do ângulo mandibular, mostrando-se um método eficaz e com baixo índice de complicações pós-operatórias e a técnica de AO/ASIF, sendo uma abordagem extraoral, que possui indicação para fraturas com grande deslocamento e maiores danos aos tecidos moles extraorais (Neto; Lopes \& Mandarino, 2018). 
O objetivo do presente estudo é analisar literatura acerca das técnicas mais eficazes para fixação diante de fraturas no osso mandibular.

\section{Metodologia}

Trata-se de uma revisão integrativa da literatura realizada no mês de setembro de 2021, através da aquisição de dados científicos da plataforma Literatura Latino-Americana e do Caribe em Ciências da Saúde (LILACS), do período de 2015 a 2021, nos idioma português e inglês.

Para elaborar essa produção científica foram seguidas seis etapas: elaboração da questão norteadora, busca em base de dados científicos, coleta de dados, análise dos dados da extração dos estudos científicos, interpretação e discussão dos resultados e apresentação da revisão integrativa da literatura (Souza, Silva \& Carvalho, 2010).

Inicialmente, o objetivo foi responder a pergunta norteadora do presente estudo que é: Quais são as principais técnicas cirúrgicas adotadas em fixação de mandíbula? Para encontrar os artigos científicos foram utilizados os seguintes descritores: Fixação de fratura; Mandíbula; Traumatismos mandibulares. Realizaram-se as buscas com os descritores de maneira individual e logo após foram feitos os cruzamentos com os descritores sem nenhum operador booleano.

Os critérios de inclusão foram artigos publicados em português e inglês, disponíveis eletronicamente na íntegra, cujo período de publicação compreendesse de 2015 a 2021 e indexados no Lilacs. Foram excluídos os trabalhos de conclusão de curso (TCC), teses, livros, dissertações, resumos, os estudos que abordavam as principais técnicas de fixação de maneira muito superficial e as produções científicas cujo idioma não fosse o português e inglês. Após a análise e seguindo os critérios de inclusão e exclusão, foram selecionados 11 artigos. Os artigos selecionados foram classificados em uma tabela (Quadro 1) e utilizados na construção do artigo cientifico.

Quadro 1. Publicações encontradas entre os anos de 2015 e 2021 segundo a base de dados Lilacs.

\begin{tabular}{|c|c|c|c|c|}
\hline DESCRITOR & $\begin{array}{c}\text { TOTAL DE } \\
\text { PUBLICAÇÕES }\end{array}$ & $\begin{array}{c}\text { PUBLICAÇÕES } \\
\text { FILTRADAS }\end{array}$ & $\begin{array}{c}\text { APÓS LEITURA DO } \\
\text { TÍTULO }\end{array}$ & APÓS LEITURA DO RESUMO \\
\hline Fixação de fratura & 1.818 & 240 & 14 & 8 \\
\hline Mandíbula & 69.172 & 720 & 8 & 6 \\
\hline $\begin{array}{c}\text { Traumatismos } \\
\text { mandibulares }\end{array}$ & 282 & 33 & 7 & 6 \\
\hline $\begin{array}{c}\text { Fixação de fratura e } \\
\text { Mandíbula }\end{array}$ & 1.166 & 22 & 2 & 2 \\
\hline $\begin{array}{c}\text { Fixação de fratura e } \\
\text { Traumatismos } \\
\text { mandibulares }\end{array}$ & 1.310 & 7 & 5 & 4 \\
\hline $\begin{array}{c}\text { Mandíbula e } \\
\text { Traumatismos } \\
\text { mandibulares }\end{array}$ & 1.287 & 19 & & 2 \\
\hline $\begin{array}{c}\text { Fixação de fratura, } \\
\text { Mandíbula e } \\
\text { Traumatismos } \\
\text { mandibulares }\end{array}$ & 318 & & & \\
\hline
\end{tabular}

Fonte: Autores. 


\section{Resultados}

Nesta revisão integrativa foram selecionados 15 (quinze artigos científicos) que atenderam aos critérios de inclusão e exclusão previamente estabelecidos, destacando ainda que 33 (trinta e três) inicialmente foram selecionados, mas após a análise pode-se observar que alguns se encontravam repetidos, uma vez que estes artigos apresentavam mais de um descritor em comum. As informações acerca dos artigos incluídos estão apresentadas no Quadro 2.

Quadro 2. Apresentação das características dos artigos incluídos na Revisão Integrativa.

\begin{tabular}{|c|c|c|c|c|}
\hline TÍTULO & AUTORES & ANO & $\begin{array}{l}\text { DELINEAMENT } \\
\mathrm{O}\end{array}$ & DESFECHO \\
\hline $\begin{array}{l}\text { Acesso extraoral para } \\
\text { tratamento cirúrgico de } \\
\text { fratura de mandíbula } \\
\text { atrófica: relato de caso }\end{array}$ & Oliveira, et al., & 2021 & Relato de Caso & $\begin{array}{l}\text { Por representar dois terços de todas as fraturas na } \\
\text { face, a mandíbula é bastante frequentemente atingida } \\
\text { por traumas. Sendo originalizada por acidentes } \\
\text { motociclísticos e automobilísticos, acidentes } \\
\text { desportivos, queda da própria altura ou agressões } \\
\text { físicas. }\end{array}$ \\
\hline $\begin{array}{l}\text { Fratura condilar em paciente } \\
\text { pediátrico: um relato de caso } \\
\text { condilar }\end{array}$ & Costa, et al., & 2021 & Relato de Caso & $\begin{array}{l}\text { Entre as fraturas na face de pacientes pediátricos, as } \\
\text { fraturas no osso mandibular são as mais comuns, } \\
\text { respondendo entre } 20 \% \text { e } 50 \% \text { do total de fraturas } \\
\text { faciais } 1 \text {, destacando que, o côndilo mandibular é a } \\
\text { área mais acometida em pacientes pediátricos. }\end{array}$ \\
\hline $\begin{array}{l}\text { Fratura de mandíbula } \\
\text { causada por projétil de arma } \\
\text { de fogo: Relato de caso }\end{array}$ & Antoniette, et al., & 2020 & Relato de caso & $\begin{array}{l}\text { Traumas que acometem a região da face podem } \\
\text { causar danos a várias estruturas anatômicas, sendo a } \\
\text { maxila e mandíbula as principais estruturas } \\
\text { acometidas por esses traumas. O diagnóstico e } \\
\text { tratamento precisam ser apropriados para que os } \\
\text { resultados funcionais e estéticos sejam satisfatórios, } \\
\text { pois quando não são tratados da forma correta podem } \\
\text { causar sequelas permanentes. }\end{array}$ \\
\hline $\begin{array}{lrr}\text { Fixação } & \text { single-point } & \text { com } \\
\text { placa reabsorvível } & \text { em } \\
\text { fratura pediátrica } & \end{array}$ & Lima, et al., & 2020 & Relato de caso & $\begin{array}{l}\text { O plano de tratamento para fraturas faciais, assim } \\
\text { como para qualquer tratamento, deverá ser sempre } \\
\text { individualizado, visto que a conduta deverá causar a } \\
\text { menor morbidade possível e proporcionar estética e } \\
\text { função adequada sem prejudicar o crescimento ósseo } \\
\text { fisiológico. }\end{array}$ \\
\hline $\begin{array}{l}\text { Osteomielite originada por } \\
\text { fratura mandibular não } \\
\text { tratada }\end{array}$ & Ciola, et al., & 2020 & Relato de caso & $\begin{array}{l}\text { A osteomielite é uma inflamação que pode ser } \\
\text { explicada pela disseminação de microorganismos por } \\
\text { meio de traumas e procediementos dentais. O } \\
\text { diagnóstico precoce dessa doença é necessário para } \\
\text { uma conduta de tratamento conservador. }\end{array}$ \\
\hline $\begin{array}{l}\text { Treatment of Resorbed } \\
\text { Mandibles with Titanium } \\
\text { Plate and immediate Implant } \\
\text { - Supported Prosthesis }\end{array}$ & Fabris, et al., & 2019 & Relato de Caso & $\begin{array}{l}\text { Reabsorção óssea mandibular severa pode fraturar no } \\
\text { decorrer ou depois da instalação de implantes, para } \\
\text { esse caso, em um único procedimento cirúrgico, pode } \\
\text { ser posicionada a placa de titânio antes de colocar os } \\
\text { implantes. }\end{array}$ \\
\hline $\begin{array}{l}\text { Fratura de Mandíbula: } \\
\text { Relato de Caso }\end{array}$ & Flandes, et al., & 2019 & Relato de Caso & $\begin{array}{l}\text { Há uma maior prevalência de fraturas mandibulares } \\
\text { em adultos do sexo masculino. Essas fraturas } \\
\text { necessitam ser diagnósticas e tratadas da forma mais } \\
\text { adequada, visando sempre o restabelecimento } \\
\text { funcional e estético do paciente. }\end{array}$ \\
\hline $\begin{array}{l}\text { Fratura de mandíbula } \\
\text { associada à transmigração de }\end{array}$ & Santos, et al., & 2019 & Relato de Caso & $\begin{array}{l}\text { Um dos fatores que pode contribuir para a ocorência } \\
\text { de uma fratura mandibular é a presença de dentes } \\
\text { iclusos, visto que podem ocasionar uma diminuição }\end{array}$ \\
\hline
\end{tabular}




\begin{tabular}{|c|c|c|c|c|}
\hline canino & & & & $\begin{array}{l}\text { óssea em determinada região e estabelecer a } \\
\text { localização e padrão da fratura, visto isso, deve-se } \\
\text { avaliar como um todo os riscos e benefícios para } \\
\text { retirada ou não do dente. }\end{array}$ \\
\hline $\begin{array}{l}\text { Fratura cominutiva de } \\
\text { mandíbula por fogos de } \\
\text { artifício. Relato de caso. }\end{array}$ & Dantas, et al., & 2018 & Relato de caso & $\begin{array}{l}\text { Os traumatismos mostram-se bastante presentes no } \\
\text { cenário da sociedade, tendo com um dos principais } \\
\text { responsáveis por afetar tanto a função quanto o } \\
\text { emocional o trauma facial. Como causas destacam-se } \\
\text { os acidentes automobilísticos e violência, no entanto, } \\
\text { outras causas como os fogos de artifícios podem } \\
\text { promover traumas perigosos. A destruição ocorrida } \\
\text { por estes fogos requer tratamento imediato e } \\
\text { multidisciplinar, além de acompanhamento durante } \\
\text { um tempo para observar e controlar riscos de } \\
\text { infecção. }\end{array}$ \\
\hline $\begin{array}{l}\text { Manejo cirúrgico de fratura } \\
\text { de mandíbula: relato de caso }\end{array}$ & Rodrigues, et al., & 2018 & Relato de Caso & $\begin{array}{l}\text { Na presença de fratura e deslocamento dos cotos sem } \\
\text { a perda de continuidade óssea, pela eficácia vista, } \\
\text { indica-se a conduta cirúrgica através do sistema de } \\
\text { fixação interna rígida, utilizando dispositivos load- } \\
\text { sharing. }\end{array}$ \\
\hline $\begin{array}{l}\text { Estudo comparativo do } \\
\text { tratamento de fraturas de } \\
\text { ângulo mandibular: análise } \\
\text { retrospectiva de sete anos }\end{array}$ & Luciano, et al., & 2018 & $\begin{array}{c}\text { Estudo } \\
\text { Observacional }\end{array}$ & $\begin{array}{l}\text { Fraturas em mandibula têm uma alta incidência no } \\
\text { atendimento buco maxilofacieal, sendo a região } \\
\text { goníaca uma das mais acometidas, podendo ser } \\
\text { indicada como a fratura mandibular com maior } \\
\text { predominância. }\end{array}$ \\
\hline $\begin{array}{l}\text { Fratura complexa de } \\
\text { mandíbula: relato de caso }\end{array}$ & Dantas, et al., & 2017 & Relato de caso & $\begin{array}{l}\text { Apesar de muito resistente, a mandíbula é a estrutura } \\
\text { que está em segundo lugar na maioria dos estudos } \\
\text { dentre as fraturas faciais. Dentre as opções de } \\
\text { tratamento a mais frequente é a abordagem cirúrgica } \\
\text { para fixação através de placas e parafusos. }\end{array}$ \\
\hline $\begin{array}{l}\text { Infant Multiple Mandibular } \\
\text { Fracture: Case Report }\end{array}$ & Claudia, et al., & 2016 & Relato de caso & $\begin{array}{l}\text { Na pediatria há uma baixa incidência de fraturas } \\
\text { faciais, no entanto, alguns casos são relatados na } \\
\text { literatura. Quando ocorrem, as fraturas afetam, } \\
\text { principalmente, a mandíbula e correspondem a fratura } \\
\text { única. Ademais, na pediatria é preciso a obtenção de } \\
\text { redução anatômica adequada e estabilidade da fratura } \\
\text { para uma boa recuperação da forma e função, } \\
\text { consequentemente, do desenvolvimento das } \\
\text { estruturas dentárias e faciais. }\end{array}$ \\
\hline $\begin{array}{l}\text { Fratura mandibular atípica } \\
\text { por arma branca: relato de } \\
\text { caso }\end{array}$ & Jínior, et al., & 2015 & Relato de caso & $\begin{array}{l}\text { Nesses casos o atendimento precisa ser o mais rápido } \\
\text { possível para precaver agravos pós-operatórios e } \\
\text { sequelas. Sendo de fundamental importância a } \\
\text { presença da equipe de CTBMF no ambiente } \\
\text { hospitalar para o tratamento adequado de pacientes } \\
\text { traumatizados em região de face. }\end{array}$ \\
\hline $\begin{array}{l}\text { Tratamento de fratura } \\
\text { complexa de mandíbula por } \\
\text { abordagem transcervical: } \\
\text { Relato de caso }\end{array}$ & Alencar, et al., & 2015 & Relato de caso & $\begin{array}{l}\text { Em casos de fraturas complexas de mandíbula com } \\
\text { múltiplos fragmentos, uma boa alternativa para o } \\
\text { tratamento seria a abordagem transcervical para } \\
\text { redução e fixação dos fragmentos ósseos através de } \\
\text { uma placa do sistema } 2.4 \mathrm{~mm} \text { do tipo locking que } \\
\text { mostrou-se previsível, seguro e com baixo índice de } \\
\text { complicação. }\end{array}$ \\
\hline
\end{tabular}

Fonte: Autores. 


\section{Discussão}

Na rotina dos cirurgiões bucomaxilofaciais a presença de fraturas que acometem a mandíbula possui uma frequência significativamente alta, afetando as estruturas anatômicas faciais, o que requer do profissional uma conduta adequada e precisa, determinando um diagnóstico e tratamento efetivos, buscando devolver ao paciente a estética e função normais (Jínior et al., 2015; Santos et al., 2019; Antoniette; Junior \& Marson, 2020). Devido ao fato de esse tipo de trauma acometer estético e funcionalmente o paciente, faz-se necessário um acompanhamento pós-operatório, prevenindo complicações como infecções e necessidade de realizar novos procedimentos cirúrgicos (Dantas et al., 2018).

É visto que os principais fatores que levam a esse tipo de trauma são acidentes de trânsito e agressões físicas (Dantas et al., 2017). Além do mais, a faixa etária mais prevalente corresponde aos adultos jovens e do sexo masculino (Flandes; Dias \& Junior, 2019). No entanto, a presença de fraturas em mandíbula pode também ocorrer, requerendo uma abordagem conservadora, visando evitar sequelas, deformidades e alguma complicação após a cirurgia (Toniolli et al., 2019; Costa et al., 2021).

Existem fraturas mandibulares complexas, que apresentam múltiplos fragmentos dos dois lados, nesses casos o tratamento de abordagem transcervical com placa do sistema $2.4 \mathrm{~mm}$ do tipo locking para redução e fixação dos fragmentos ósseos, mostrou ser seguro e com baixo índice de complicação. (Alencar et al., 2015). Em casos de tratamento de fratura de ângulo mandibular a maior parte das fraturas foi fixada com 2 miniplacas, utilizando a técnica de Champy em alguns dos pacientes, o que não houve diferença no índice de complicações e no sucesso do tratamento. Geralmente o tratamento utilizado depende da preferência do profissional. (Luciano et al., 2018)

O acesso extraoral para tratamento de fratura mandibular promovendo fixação interna e redução aberta mostrou ser favorável, junto com abordagem submandibular proporcionando um bom acesso, com boa visão do campo operatório. (Oliveira et al., 2021). Atualmente, existe um tratamento totalmente seguro, com a utilização de placa de titânio, a qual pode ser fixada na mandíbula reabsorvíveis com osso cortical remanescente, assim, evita obstáculos biomecânicos no procedimento com próteses totais imediatas implantosuportadas. (Fabris et al., 2019).

O trauma facial é um evento comum na população, constituindo um procedimento corriqueiro e de frequência crescente para os profissionais cirurgiões buco-maxilo-faciais, constituindo um sério problema de saúde pública e adicionalmente causam deformidades e/ou risco importante à vida. Uma fratura na mandíbula não passa jamais despercebida, pois é bastante dolorosa que se amplifica com os movimentos mastigatórios, articulatórios e, até movimentos respiratórios e, por vezes, com queixas de assimetrias faciais (Silva et al., 2019). Traumas na região da face, tratamento inadequado de uma fratura, ou irradiação na mandíbula podem predispor a doenças, como a osteomielite (Ciola et al., 2020).

Apesar da mandíbula ser uma estrutura óssea bastante resistente ela está exposta a ocorrência de traumas direto ou patológicos. A segunda maior causa de traumas na mandíbula é causada por arma de fogo, durante seu tratamento o projétil só poderá ser removido quando estiver localizado de forma superficial ou caso esteja causando alguma limitação funcional. (Antoniette et al., 2020; Lima et al., 2020).

Existem diferentes padrões de fraturas mandibulares podendo ser classificadas por diversos aspectos, como os tipos, simples ou fechada que não apresenta nenhum contato com o meio externo, composta ou aberta que são fraturas que apresentam comunicação direta com o meio externo devido à incisão dos tecidos, galho verde que há uma descontinuidade incompleta do fragmento ósseo, cominutivas que são fraturas nos quais o osso apresenta diversos fragmentos ósseos (Marino; Bianchi 2020; Jesus, 2021). As fraturas de mandíbula podem, também, ser divididas em favoráveis ou desfavoráveis, podendo ser classificadas quanto sua localização, configuração e de acordo com o seu deslocamento. As fraturas favoráveis são aquelas que não ocorrem deslocamento significativo ósseo por força ação muscular, encontrando-se em sua respectiva localização. Já 
as desfavoráveis ocorrem quando há movimentação dos fragmentos ósseos por ação dos músculos (Flandes et al., 2019; Flandes et al., 2019).

Os principais exames radiográficos solicitados para pacientes com fratura mandibular são as radiografarias lateral oblíqua de mandíbula direita ou esquerda, póstero-anterior de mandíbula (towne), towne reversa e panorâmica. A tomografia computadorizada, alternativa bastante utilizada, é o exame de imagem mais completo e que apresenta, ao profissional, uma visão tridimensional acerca das fraturas, possibilitando, então, um diagnóstico mais preciso (Bermejo et al., 2016).

O tratamento cirúrgico das fraturas em face objetiva restabelecimento da função, estética e anatomia da região com auxílio de miniplacas e parafusos metálicos para esse fim. A adequada cicatrização ocorre graças à reaproximação dos cotos e à sua imobilidade, estabelecida pelo sistema de fixação interna rígida. Os dispositivos load-bearing são miniplacas e parafusos do sistema $2.4 \mathrm{~mm}$ a $3.0 \mathrm{~mm}$, que absorvem toda a carga gerada sobre o osso, no intuito de evitar maior deslocamento e instabilidade da fratura. Os dispositivos load-sharing consistem em miniplacas e parafusos do sistema $1.5 \mathrm{~mm}$ a $2.0 \mathrm{~mm}$, que buscam o compartilhamento das cargas geradas sobre o osso fraturado. Quando se refere aos sistemas de fixação Interna rígida em cirurgia e traumatologia bucomaxilo-facial, dois dispositivos podem ser utilizados. (Rodrigues, et al., 2018).

\section{Considerações Finais}

É possível que as fraturas mandibulares estejam presentes no dia a dia do cirurgião-dentista, aparecendo com frequência na rotina dos bucomaxilofaciais e necessitando de conhecimentos para realização de seu tratamento. São ocasionadas por traumas como, por exemplo, acidente e agressão física. Dessa forma, as técnicas de fixação são as opções de intervenção, as quais utilizam miniplacas e parafusos metálicos, destacando-se a técnica de Champy com alto índice de sucesso. O objetivo das técnicas de fixação é reestabelecer a função, estética e anatomia da área afetada. Ademais, é imprescindível o acompanhamento pós-operatório para prevenção de complicações.

\section{Referências}

Alencar, M. G. M., Rebelo, H. L., Júnior, E. Z. S., Júnior, M. A. B., \& Júnior, M. D. M. (2015). Tratamento de fratura complexa de mandíbula por abordagem transcervical: Relato de caso. Rev. cir. traumatol. buco-maxilo-fac. 15(4), 43-48.

Antoniette, R. N., Junior, J. C. R., \& Marson, G. B. O. (2020). Fratura de mandíbula causada por projétil de arma de fogo: Relato de caso. Rev. Cir. Traumatol. Buco-Maxilo-Fac. 20(4), 35-39.

Arantes, E. R., Silva, F. B. M., Corrêa, R. M., \& Sartoretto, S. C. (2019). Tratamento cirúrgico de fratura cominutiva de mandíbula: relato de caso. International journal of science dentistr, $51,52-61$.

Bermejo, P. R., Coléte, J. Z., Momesso, G. A. C., Oliveira, P. A., Fonseca, J. H. \& Shinohara, E. H. (2016). Tratamento cirúrgico de fratura mandibular decorrente de projétil de arma de fogo: relato de caso. Archives of Health Investigation. 5(6).

Ciola, P., Gross, D. J., Andreis, J. D., Yokoyama, P. J. I., Santos, M. G. \& Martins, L. (2020). Osteomielite originada por fratura mandibular não tratada. Rev. cir. traumatol. buco-maxilo-fac. 20(3), 29-33.

Claudia, L., Matilde, L., Lucinéia, B., Rodolfo, O. \& Roberto, P. (2016). Infantmultiple mandibular fracture: case report. Int. j. med. surg. sci. 3(2), 823-7.

Costa, C. F. B., Gusmão, T. B., Loureiro, C. E., Pereira, L. De A., Galvão, A. C., \& Lucindo Sobrinho, A. (2021). Fratura condilar em paciente pediátrico: um relato de caso condilar. Rev. Odontol. Araçatuba, 4(3), 16-20.

Dantas, A. C. G. C., Silva, T. P., Silva, J. C., Aragão, E. S., Rocha, R. S., \& Santos, M. A. M. (2018). Fratura cominutiva de mandíbula por fogos de artifício. Relato de caso. Rev. Cir. Traumatol. Buco-Maxilo-Fac., 18(3), 17-21.

Dantas, B. P. S. S., Fialho, P. V., Fernandes, A. C. F., Silva, D. T., \& Queiroz, C. S. (2017). Fratura complexa de mandíbula: relato de caso. Revista odontológica de Araçatuba. 38(3), 43-48.

Fabris, V., Reginato, V. F., Smaniotto, C., Bacchi, A., \& Consani, R. L. X. (2019). Treatment of Resorbed Mandibles with Titanium Plate and immediate Implant - Supported Prosthesis: relato de caso. Brazilian dental journal. 30(3), 244-251. 
Research, Society and Development, v. 11, n. 1, e30511124821, 2022

(CC BY 4.0) | ISSN 2525-3409 | DOI: http://dx.doi.org/10.33448/rsd-v11i1.24821

Flandes, M. P., Galvão, L. B., \& Júnior, W. P. (2019). Fratura de mandíbula: estudo epidemiológico de 93 casos. Braz. J. Hea. Ver. 2(5), $4427-4435$.

Flandes, M., Dias, L., \& Paulesini Junior, W. (2019). Fratura de mandíbula - relato de caso. Revista de Odontologia da Universidade Cidade de São Paulo, 31(2), 205-212.

Jínior, E. F. S., Dias, T. G. S., Barbalho, J. C. M., Moraes, D., \& Morais, H. H. A. (2015). Fratura mandibular atípica por arma branca: relato de caso. Rev. cir. traumatol. buco-maxilo-fac. 15(3), 87-91.

Jesus, B. B. da S., Santos, A. K. L. dos., Silva, K. S. da., Gromatzky, P. R. ., \& Seroli, W. (2021). Fraturas mandibulares. E-Acadêmica, 2(3), 1-7.

Lima, L. H. F., Cesconetto, L. A., Nogueira, L. H. M., Vilas Boas, J. L., Albuquerque Neto, A. D., \& Oliveira, É. M. F. (2020). Fixação single-point com placa reabsorvível em fratura pediátrica. Relato de caso. Brazilian Dental Journal. 30(3) 244-251.

Luciano, A. A., Garbin-Júnior, E. Á., Magro-Érnica, N., Griza, G. L., Oliveira, G. R., \& Conci, R. A. (2018). Estudo comparativo do tratamento de fraturas de ângulo mandibular: análise retrospectiva de sete anos. Rev. cir. traumatol. buco-maxilo-fac , 18(3), 10-16.

Marino, P. A. M., Bianchi, T. (2020). Fratura de sínfise mandibular: uma revisão literária sobre incidência, tipos de fratura e formas de tratamento. Revista Interciência, 1(4), 25-28.

Neto, O. B., Lopes, M. F., \& Mandarino, S. C. A. (2018). Tratamento de Fratura Mandibular Pela Técnica de Champy: Relato de Caso. Brazilian Journal of Surgery and Clinical Research, 24(3), 101-104.

Oliveira, G. P., Carvalho, M. M. M., Rodrigues, R. D., Barbosa, D. M. O., Borges, E. F. D., \& Seixas, A. M. (2021). Acesso extraoral para tratamento cirúrgico de fratura de mandíbula atrófica: relato de caso. Rev. Odontol. Araçatuba, 42(2), 42-46.

Rodrigues, R., Quintas, P., Barreto, L., Costa, C., Silva, L., \& Aguiar, J. (2018). Manejo cirúrgico de fratura de mandíbula: relato de caso. Revista Da Faculdade De Odontologia - UPF, 23(3), 343-347.

Santos, E. A., Costa, C. F. B., Quintas, P. H., Dultra, J. A., \& Dultra, F. K. A. (2019). Fratura de mandíbula associada à transmigração de canino. Rev. Cir. Traumatol. Buco-Maxilo-Fac., 19(4), 29-33.

Silva, N. K. S., Marques, A. L., \& Almeida-Marques, R. V. D. (2019). Perfil das fraturas faciais em um serviço de emergência no Maranhão. Rev. cir. traumatol. buco-maxilo-fac, 19(1), 8-13. 